\title{
Plastic anisotropy of textured titanium sheets
}

\author{
V.N. Serebryany, ${ }^{1}$ J.-J. Fundenberger, ${ }^{2}$ K.E. Mel'nikov, ${ }^{1}$ F. Wagner ${ }^{2}$ \\ ${ }^{1}$ A.A. Baikov Institute of Metallurgy and Material Science, RAS, Leninsky pr., 49, \\ 119991 Moscow, Russia \\ ${ }^{2}$ Paul Verlaine University of Metz, Ile du Saulcy, 57045 Metz, Cedex 1, France \\ vns@ultra.imet.ac.ru
}

Formability of the titanium sheets essentially depends on a normal anisotropy parameter $(R)$ of the investigated material. The magnitude of the $R$-parameter was defined from orientation distribution function (ODF) data in the context of the suggested plastic deformation model ${ }^{1,2}$. In the present paper we study influence of texture on the normal plastic anisotropy parameter $R$ for example of Ti sheet samples ${ }^{3}$.

\section{Method of plastic anisotropy prediction}

To estimate the effects of texture on $R$ parameter $M$ - factors were calculated based on the Taylor-Bishop-Hill model. The mean value of the Taylor $M$-factor is given by

$$
M(q)=\int M(q, g) f(g) d g
$$

where $q$ is the contraction ratio, $f(g)$ is ODF function. By expressing $M(q, g)$ and $f(g)$ in the form of spherical harmonics and substituting these formulas into Eq. (1) it can be found that

$$
M(q, \alpha)=\sum_{l} \sum_{\mu} \sum_{v} \frac{m_{l}^{\mu v}(q) C_{l}^{\mu v}}{2 l+1} \cos (v \alpha)
$$

where $\alpha$ is the angle between the tensile axis and the rolling direction; $m_{l}^{\mu v}(q)$ and $C_{l}^{\mu v}$ are the coefficients of the expansion $M(q, g)$ and $f(g)$ into a series of the spherical harmonics. If a sample is elongated in a free tensile experiment then elongation is fixed by the experimental conditions but not the contraction ratio $q$. It will take on such a value that the deformational work is minimum which requires

$$
\frac{\partial M(\bar{q}, \alpha)}{\partial q}=0
$$

The solution of Eq. ( 3 ) with Eq. ( 2 ) yields $q=q_{\min }(\alpha)$ which is assumed to be the actual contraction ratio related to the normal anisotropy coefficient $R$ by

$$
R(\alpha)=\frac{q_{\min }(\alpha)}{1-q_{\min }(\alpha)}
$$

The basic assumptions involved in this method for relating texture and normal anisotropy coefficient are that:

(i)The series expansion method gives an adequate description of the experimental textures;

(ii)The Taylor - Bishop - Hill theory can be used as reasonable model for the tensile deformation;

(iii) $q_{\text {min }}$ can be used as a measure for the $R$ of the material.

The aim of this work is to prove the validity of these assumptions as applied to Ti sheets.

\section{Materials and techniques of measure and calculation}

The two types of the commercially pure titanium sheets with thickness $0,9 \mathrm{~mm}$ were used in the recrystallized condition after cold rolling with the different concentrations of the interstitial impurities $\mathrm{O}$ and $\mathrm{N}$ : the first type (t1) of the sheets had $2500 \mathrm{ppm} \mathrm{O}$ and $100 \mathrm{ppm}$ $\mathrm{N}$, and the second type $(\mathrm{t} 2)$ of ones had $500 \mathrm{ppm} \mathrm{O}$ and $400 \mathrm{ppm} \mathrm{N}$. The plastic anisotropy is 
estimated by the $R$ coefficient equal a ratio of lateral to normal strains in the sheet plane after $5 \div 10 \%$ elongation (the limit of homogenous strain for these materials). This anisotropy parameter of the sheet material was evaluated using uniaxial tensile tests at Instron machine with the strain velocity $0,002 \div 0,003 \mathrm{~s}^{-1}$ at room temperature. The tensile samples were cut from the titanium sheets at the different $\alpha$ - angles to rolling direction. The texture analysis was based on pole figure measurement of the $t 1$ and $t 2$ sheets, carried out at the two laboratories: LETAM, Paul Verlaine University(Metz, France) and SSL, Baikov Institute (Moscow, Russia). The texture of the $\mathrm{t} 1$ sheet was measured after $10 \%$ tensile deformation at a X-ray Siements D5000 texture diffractometer with use the Co- $K_{\text {alpha }}$ radiation. There were measured four incomplete pole figures $\{10.0\},\{00.2\},\{10.1\},\{10.2\}$ by the "on reflection" method. The range of tilt angles $\alpha\left(1.25 \div 73.75^{\circ}\right)$ and azimuth angles $\beta\left(0 \div 360^{\circ}\right)$ with step on $\alpha=2,5^{\circ}$ and $\beta=5^{\circ}$. The texture of the $\mathrm{t} 2$ sheet was measured after $5 \%$ tensile deformation at a X-ray DRON -7 texture diffractometer with use the $C u$ - $K_{a l p h a}$ radiation. There were measured six incomplete pole figures $\{10.0\},\{00.2\},\{10.1\},\{10.2\}$, $\{10.3\},\{11.0\}$ by the "on reflection" method. The range of tilt angles $\alpha\left(0 \div 70^{\circ}\right)$ and azimuth angles $\beta\left(0 \div 360^{\circ}\right)$ with step on $\alpha=2$ and $\beta=2^{\circ}$. ODF restitution was carried out by the modified harmonic method of ridge estimations ${ }^{4}$ with $L_{\max }=16$ and regularization parameter $\lambda=0,01$. This approach is an improvement of the harmonic method of texture analysis in which the ODF is represented by a sum of hyperspherical functions. The unknown even coefficients $\boldsymbol{C}$ can be defined from the system of equations represented in matrix form $p=A C+v$, where $p$ are the pole densities measured in diffraction experiment, $A$ is the system matrix and $v$ are random errors of measurement. It is very difficult to get precise results using this system of equations because the matrix $A$ is non-orthogonal (due to experimental data insufficiency and the random errors). For the solution improvement of similar problems, the statistical method of ridge estimators is offered. It is a generalization of the well known least squares method. The solution of the system of equations is searched as the smoothest function

$$
\tilde{\mathbf{n}}=\left(\mathbf{A}^{T} \mathbf{A}+\lambda \mathbf{Q}\right)^{-1} \mathbf{A}^{T} \mathbf{p}
$$

where $\lambda$ is a parameter of regularization, $\mathbf{Q}$ is a stabilizing functional, a type of which was defined by the different orders derivatives of the texture function norm ${ }^{4}$. Only terms of even rank in $L$ were considered. This causes some loss in precision of the calculated ODF. However, due to symmetries in the single crystal properties, the macroscopic sample properties, like the Taylor factor, are independent of the odd terms. Moreover the terms with a rank higher than 10 have practically no effect on the result. ODF coefficient errors are defined as diagonal elements of correlation matrix

$$
D(\hat{c})=\sigma^{2}\left(A^{T} A+\lambda Q\right)^{-1}
$$

here $\sigma^{2}$ is a dispersion of the primary experimental errors. The plastic deformation under the axial loading of the polycrystalline titanium was supposed to caused by a basal, prism and pyramidal slip of dislocations along $<1120>$ direction and by $\{1012\}<1011>$ and $\{1122\}<1123>$ twinning to provide for $c$ - axis extension and compression strain. The deformation system CRSS values were defined with according to the temperature, the strain rate and the oxygen and nitrogen contents of the titanium from the previously obtained results. The CRSS values of the activated slip and twinning systems for titanium sheets $\mathrm{t} 1$ and t 2 are presented in Table 1. For the simulation of the plastic deformation under the axial loading we applied Taylor model in the modification of the maximum work procedure of Bishop and Hill, being all possible stress states which can simultaneously operate at least five independent 
Table1. CRSS values for the main deformation systems of titanium of the different grades

\begin{tabular}{|c|c|c|c|c|c|}
\hline \multirow{2}{*}{ Type } & \multicolumn{5}{|c|}{$\tau, M P a$} \\
\cline { 2 - 6 } & $\{1010\}<1120>$ & $\{0002\}<1120>$ & $\{1011\}<1120>$ & $\{1012\}<1011>$ & $\{1122\}<1123>$ \\
\hline $\mathrm{t} 1$ & 109 & 99 & 99 & 240 & 125 \\
\hline $\mathrm{t} 2$ & 54 & 98 & 71 & 196 & 196 \\
\hline
\end{tabular}

deformation systems we calculated using the Thornburg and Piehler technique ${ }^{5}$. For a given $q$-value the magnitude $M(q, g)$ is defined by a ratio the maximum for given grain orientation deformational work normalized to an unit strain to CRSS-value of the prism slip system. Then the analogous procedures were repeated for the different orientations $g=\left\{\varphi_{1}, \Phi, \varphi_{2}\right\}$, (where $\varphi_{1}, \Phi, \varphi_{2}$ are the Euler angles) varied through $0 \leq \varphi_{1} \leq 90^{\circ}, 0 \leq \Phi \leq 90^{\circ}, 0 \leq \varphi_{2} \leq 60^{\circ}$ ranges with $3^{\circ}$ steps for an each angle and 0.1 for $q$-value $(0<q<1)$. As a result, we apply the normalized $M(q, g)$ orientation functions. Then, using the above-described procedure we defined the angle dependence of the normal anisotropy coefficient in titanium sheets, being the ODF coefficients have been calculated by the modified harmonic method from pole figures. Relying on the above-described algorithm we developed the special computer programs.

\section{Calculated and experimental results}

The $\{00.2\}$ pole figures for the $\mathrm{t} 1(\mathrm{a})$ and $\mathrm{t} 2(\mathrm{~b})$ titanium sheets are presented in Fig. 1.

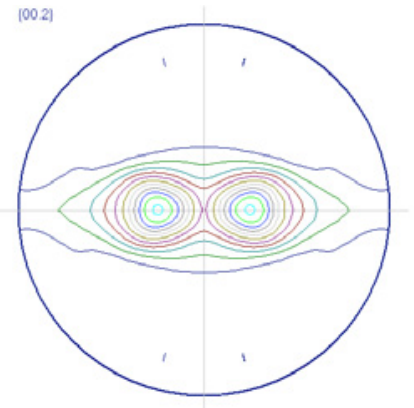

a

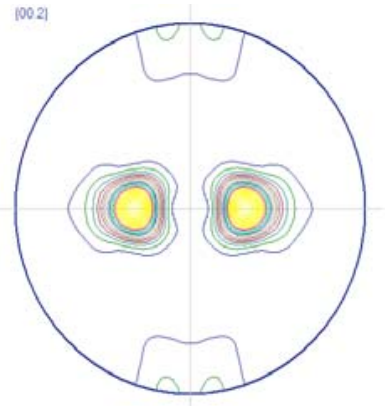

b

Fig.1. The $\{00.2\}$ pole figures for the $\mathrm{t} 1(\mathrm{a})$ and $\mathrm{t} 2(\mathrm{~b})$ titanium sheets.

Errors of ODF coefficients have maximums at the certain numbers of expansion, still the common error level is insignificant for the both cases. As a whole, the values of these errors are greater for $\mathrm{t} 1$ sheets than for $\mathrm{t} 2$ ones. This error difference is determined by the different texture plans used for the ODF restitution of the $t 1$ and $t 2$ sheets. The influence of the ODF coefficient errors on the calculated dependence of $R$ was analysed. The calculated $R(\alpha)$ values very well agree with the experimental ones (see Fig.2 and Fig.3) for the both titanium sheets. The ODF coefficient errors weakly effect on the accuracy of the calculated angle dependence of normal plastic anisotropy parameter for the both titanium sheets. Check of $q_{\text {min }}$ as a measure for the $R$ - evaluation of the material is presented in Table 2 . It is seen that $q_{\min }$ can be used as a measure for the $R$ - evaluation of the titanium sheets.

The problem of the plastic anisotropy prediction from texture data has the two main aspects: the adequate accuracy of the restituted ODF coefficients from pole figures and the adequate deformation model. As for the first aspect, the selected texture measurement plan and the modified harmonic method of ridge estimations ${ }^{4}$ secure the minimization of the ODF coefficient errors, as it is showed in the present report. In that event the first assumption (i) is 
satisfied. As for the second aspect, the calculation of normal plastic anisotropy parameter $R$ in the terms of the Taylor-Bishop-Hill model shows the very good coincidence of the results with the experimental ones. In this case the account of ODF coefficient errors during the calculation don't vary practically these results. Here, the use of criterion of the $M(q)$ minimum for the $R$ definition also fully is justified. In this case assumptions (ii) and (iii) are satisfied too.

Table.2. Values $q_{\min }$ and $q_{\text {exp }}$ obtained for titanium sheets of the different grades

\begin{tabular}{|c|c|c|c|c|c|c|c|}
\hline \multirow{2}{*}{ Type } & \multicolumn{3}{|c|}{$\mathrm{t}$. } & \multicolumn{3}{c|}{ t2 } \\
\hline$\alpha^{0}$ & 0 & 30 & 60 & 90 & 0 & 45 & 90 \\
\hline$q_{\min }$ & $\mathbf{0 , 7 6}$ & $\mathbf{0 , 8 1}$ & $\mathbf{0 , 8 6}$ & $\mathbf{0 , 8 5}$ & $\mathbf{0 , 7 1}$ & $\mathbf{0 , 8 2}$ & $\mathbf{0 , 8 7}$ \\
\hline$q_{\text {exp }}$ & $\mathbf{0 , 7 5}$ & $\mathbf{0 , 7 9}$ & $\mathbf{0 , 8 6}$ & $\mathbf{0 , 8 6}$ & $\mathbf{0 , 7 3}$ & $\mathbf{0 , 8 2}$ & $\mathbf{0 , 8 7}$ \\
\hline
\end{tabular}

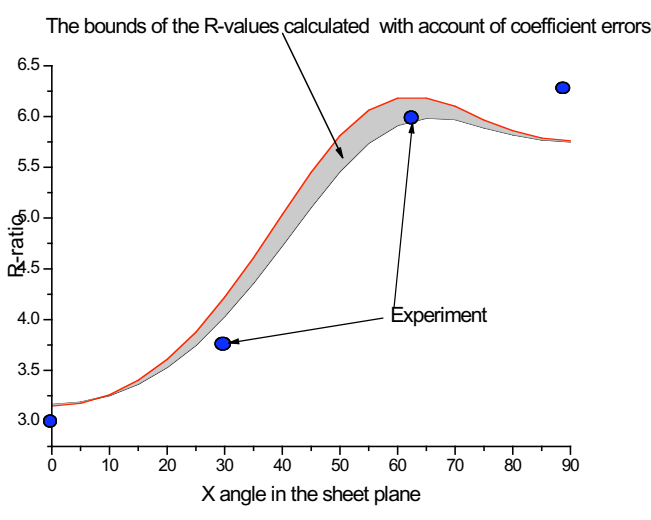

Fig.2. The calculated (lines) and experimental (points) $R(\alpha)$ values of the $\mathrm{t} 1$ titanium sheet.

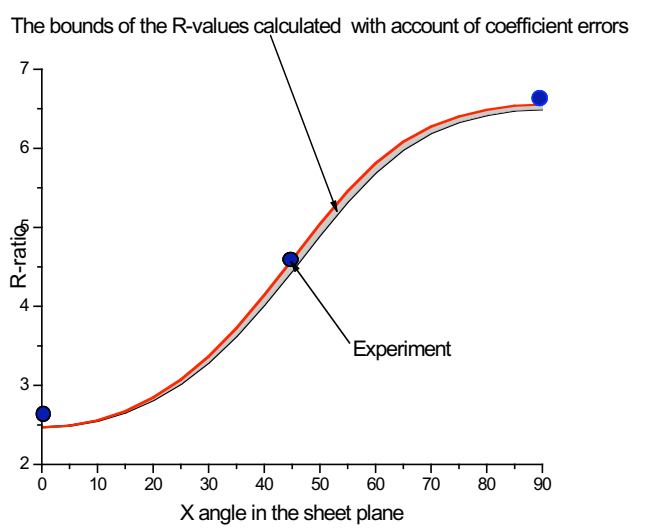

Fig.3. The calculated (lines) and experimental (points) $R(\alpha)$ values of the $\mathrm{t} 2$ titanium sheet.

\section{Conclusion}

-The optimal texture measurement plan and use of the harmonic method of ridge estimations enable to minimize the ODF coefficient errors.

-The calculation of normal plastic anisotropy parameter in terms of the Taylor-Bishop-Hill model gives the good coincidence with the experimental data as applied to Ti-sheets with the different concentrations of the interstitial impurities $\mathrm{O}$ and $\mathrm{N}$.

-The obtained results are lasting as applied to the ODF coefficient errors.

This work was supported by the INTAS under the grant № 03-51-6092.

1. H.J.Bunge, Mean Values of Physical Properties, in Quantitative Texture Analysis, DGM, Oberursel, 383 (1982).

2. H.J.Bunge, Technological Applications of Texture Analysis, Z. Metallkde 76, 457 (1985).

3. V.N. Serebryany, R.G. Koknaev, Anisotropy Parameter Evaluation in Textured Titanium Alloy Sheets, Proc. of 9th World Conf. On Titanium, 698 (2000).

4. V. N. Serebryany, S. F. Kurtasov, T.I. Savyolova, Pole Figure Measurement Plan Influence on Accuracy ODF Coefficients Determined by Modified Harmonic Method, Proc. of ICOTOM 14. Materials Science Forum, 495-497, 1693 (2005).

5. D.R. Thornburg and H.R. Piehler, An Analysis of Constrained Deformation by Slip and Twinning in Hexagonal Close Packed Metals and Alloys, Metallurgical Transactions, , 6A, 1511 